\title{
FEMORAL OSTEOTOMY IN EXTRA-ARTICULAR ARTHRODESIS OF THE TUBERCULOUS HIP
}

\author{
J. A. Cholmeley, Stanmore, England \\ From the Institute of Orthopaedics, Royal National Orthopaedic Hospital, London
}

The usual end-result of uncomplicated tuberculous disease of a joint is fibrous ankylosis. This particularly applies to the hip. With the discovery of antibiotics having a specific action on the tubercle bacillus it was hoped that a rapid and permanent cure of the disease might be obtained. It was also hoped that a mobile joint might be the common end-result and fibrous ankylosis the rarity. So far this desirable state of affairs has not materialised. This is due in part to the inability of the specific drugs to obtain access to the organism on account of the normal local reaction of the body to the disease. It is also often due to delay in diagnosis, so that bone and cartilage damage has occurred before specific drug treatment is started.

There is no doubt, however, that more rapid healing of tuberculous lesions of the hip and knee has been obtained under the influence of streptomycin and other specific drugs. It is also true that a higher percentage of mobile tuberculous joints has followed specific antibiotic treatment (Stevenson 1954). However, it seems probable that some of these mobile joints, on account of intra-articular damage, will give rise to trouble in the future.

For many years the best method of obtaining bony ankylosis of a quiescent tuberculous hip with fibrous ankylosis had been a surgical problem, and it still is. Many methods have been tried, as evidenced by the large amount of literature on the subject. It will be noted that, of the exponents of extra-articular arthrodesis, Brittain was the first to describe, in his ischio-femoral arthrodesis, the simultaneous combination of an extra-articular bone graft with osteotomy of the femur (Brittain 1941, 1942, 1948). Trumble first described the ischio-femoral bone graft but without osteotomy (Trumble 1932, 1937, Van Gorder 1949). Some form of upper femoral osteotomy alone has been used by many surgeons in an attempt to obtain bony ankylosis across the tuberculous hip, but the results have always been uncertain (Wilkinson 1947). Nevertheless the strong fibrous ankylosis often obtained by this procedure can give an excellent functional result in many cases, though it often allows deformity to occur subsequently.

Milch (1947) stated: " in the quiescent phase the likelihood of successful fusion is much greater than in the active phase ... In this (quiescent) phase the primary effect of arthrodesis may in itself be nothing more than that of enforcing rest upon which the normal processes of repair depend." An intertrochanteric or subtrochanteric osteotomy of the femur alone facilitates this rest, and when combined with an extra-articular bone graft the degree of rest at the damaged joint is even greater. This combination can be carried out simultaneously, as in Brittain's operation, or the osteotomy may be performed a short time after the grafting operation, without interfering with the immobilisation.

Not infrequently an extra-articular bone graft alone results in failure. In many such cases a femoral osteotomy, done primarily to correct deformity at the hip, will produce the desired result of bony ankylosis across the joint with consolidation of the graft (Figs. 1 to 3).

\section{CLINICAL MATERIAL}

In this paper I propose to give the results obtained at the Royal National Orthopaedic Hospital of two methods of extra-articular arthrodesis of the quiescent tuberculous hip with 
fibrous ankylosis. These two methods are the ilio-femoral graft and the ischio-femoral graft. Between 1933 and 1953, 147 such operations were carried out.

In the ilio-femoral technique the graft is obtained either from the ilium-preferably full thickness-or from the tibia. Frequently such a grafting operation has been followed by an osteotomy of the upper end of the femur. This has been done either within a few months of the grafting operation to correct a pre-existing deformity, or at a considerably later date to correct subsequent deformity which has occurred as a result of failure of the grafting operation.

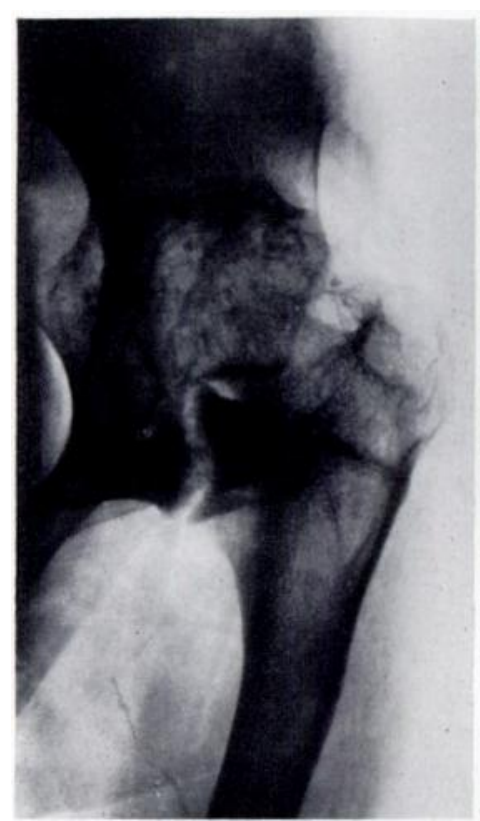

Fig. 1

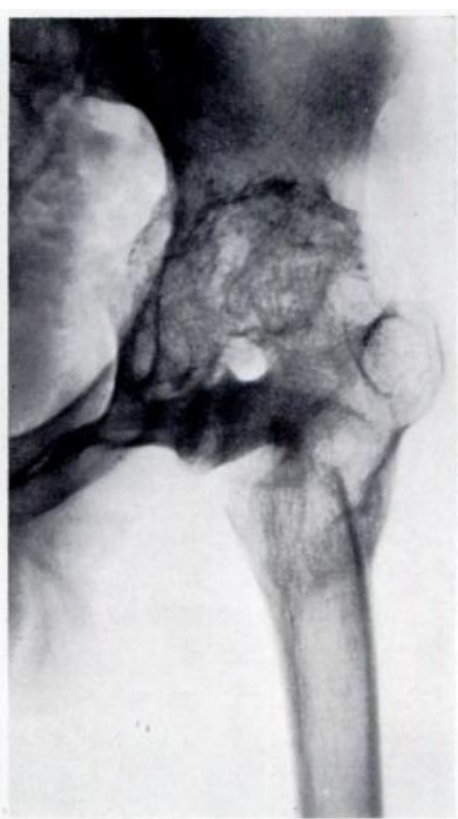

FIG. 2

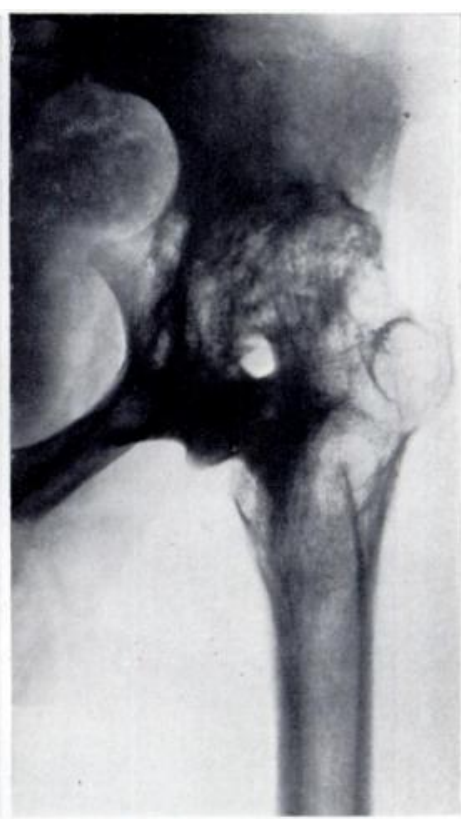

Fig. 3

Case 1-Tuberculosis of the right hip in a boy. Figure 1-Radiograph at age thirteen, showing failed ischio-femoral graft (posterior approach). Figure 2-Three months after subtrochanteric osteotomy there is union of the graft. Figure 3-Eight months after osteotomy there is bony fusion across the joint.

In the ischio-femoral method the graft has been combined as a routine with an osteotomy of the femur as described by Brittain and later modified by Foley (Foley 1949, Cholmeley and Nangle 1951).

Apart from the type of arthrodesis there are certain other factors to consider, such as the age of the patient at the time of operation. It has been found that the ideal age for any form of extra-articular arthrodesis of the tuberculous hip is between eleven and twenty years. Before eleven years satisfactory results can be obtained but less frequently than in the ideal age group. It is also necessary to immobilise and splint these young patients for a much longer period than usual (Figs. 4 to 9).

\section{RESULTS}

Of the 147 operations, fifty-five were ilio-femoral grafts without osteotomy, and thirty-seven were ilio-femoral grafts followed by an osteotomy either within a few months to correct a pre-existing deformity or at a much later date to correct a deformity caused by failure of the grafting operation. Fifty-five patients were treated by an ischio-femoral graft combined with an osteotomy, as in the Brittain or Foley type of operation. 


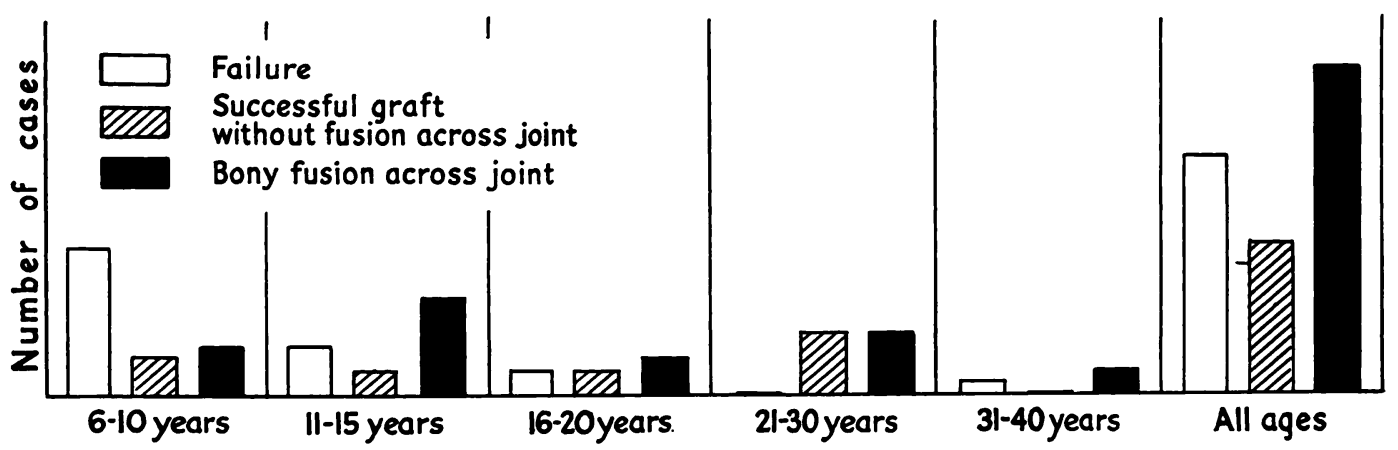

Fig. 4

Results of ilio-femoral graft without osteotomy.

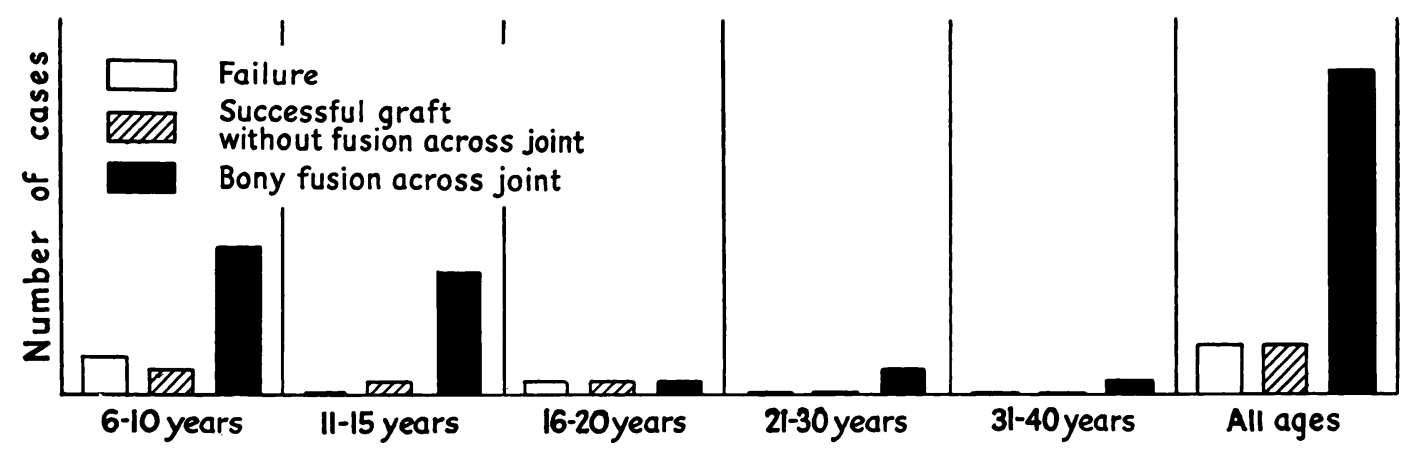

Fig. 5

Results of ilio-femoral graft with osteotomy.

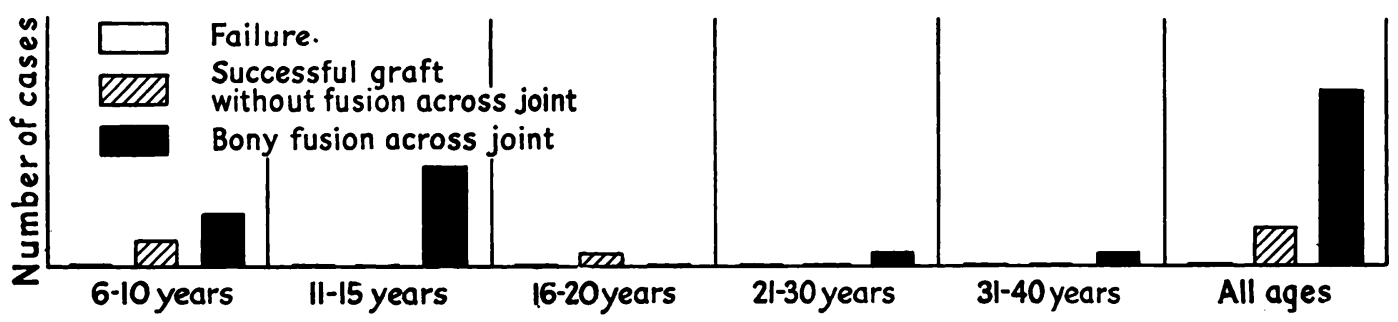

Fig. 6

Results of ilio-femoral graft with " early" osteotomy.

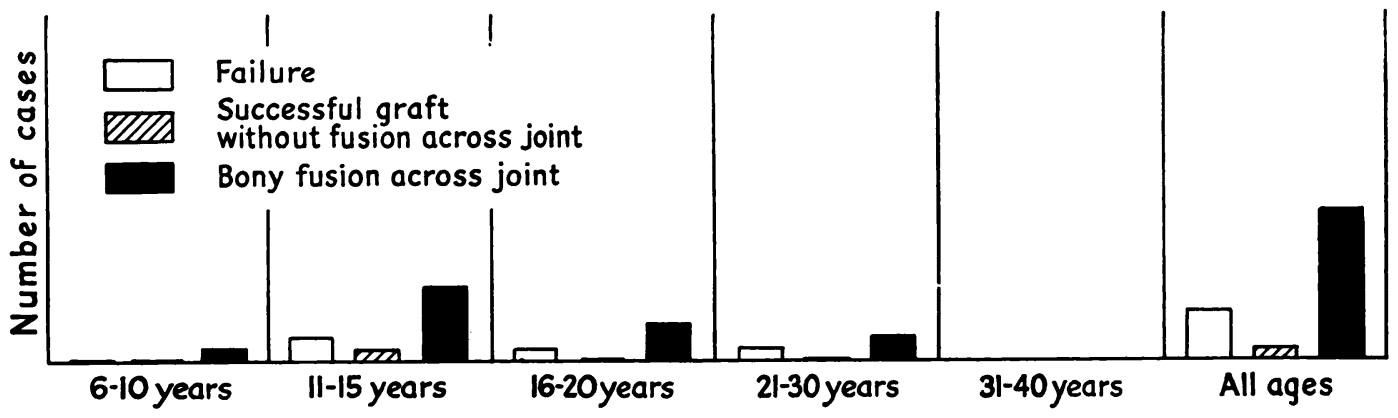

Fig. 7

Results of ilio-femoral graft with " late" osteotomy, related to age at time of osteotomy. In these cases the graft alone had failed.

THE JOURNAL OF BONE AND JOINT SURGERY 


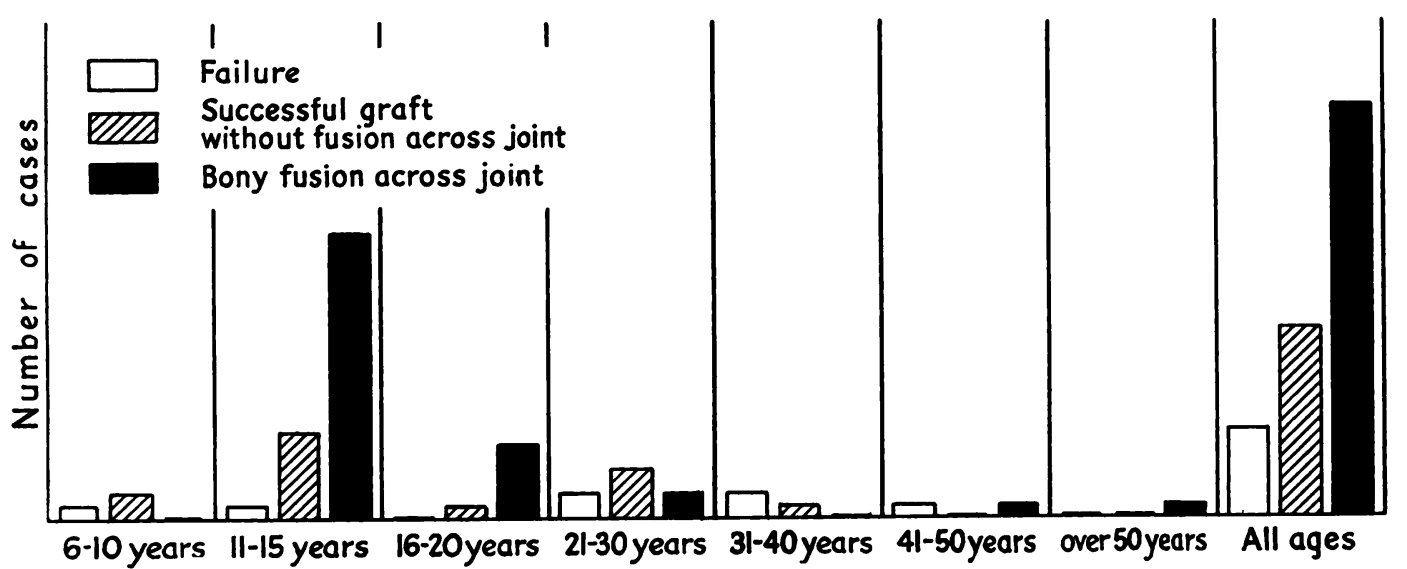

Fig. 8

Results of ischio-femoral graft.

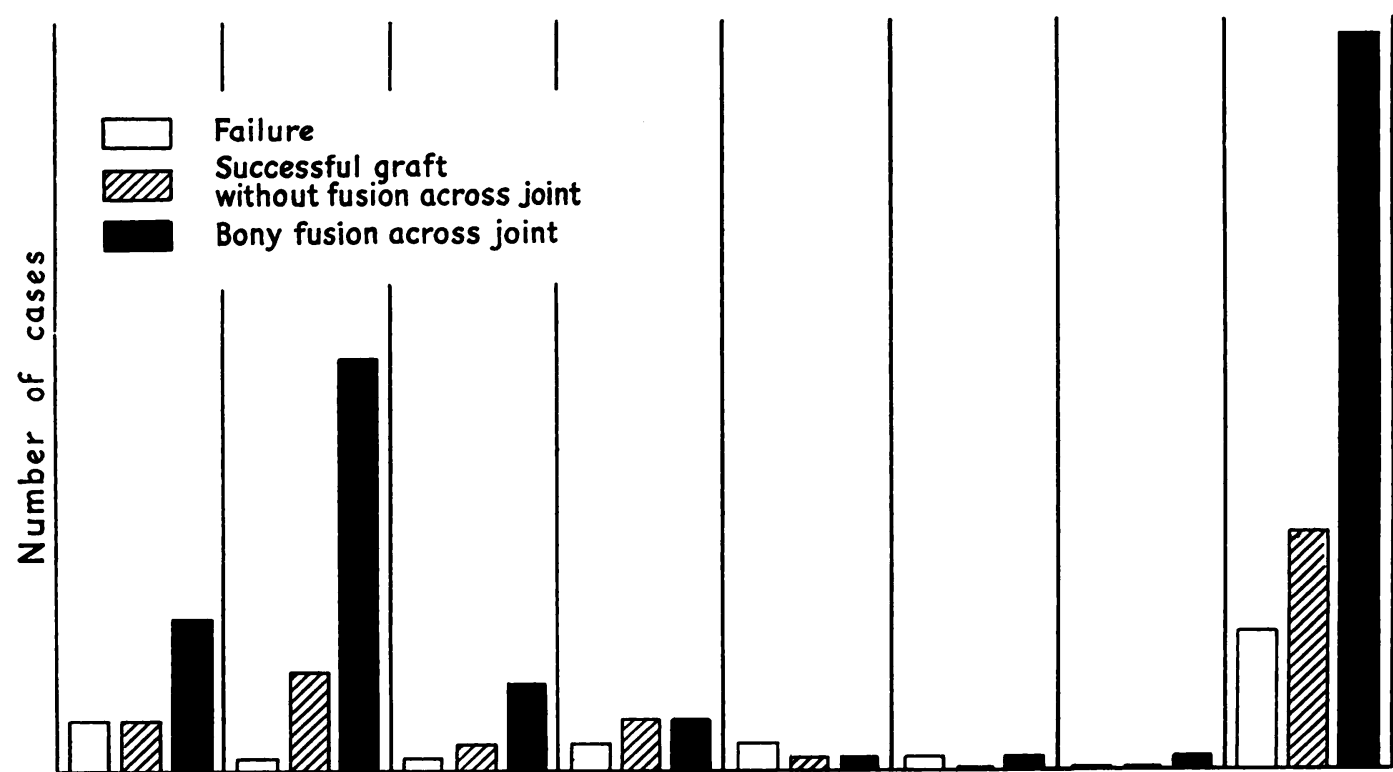

6-10 years $11-15$ years $16-20$ years $21-30$ years $31-40$ years $41-50$ years over 50 years All ages

Fig. 9

Results in all cases of ilio-femoral and ischio-femoral arthrodesis combined with osteotomy.

Of these operations four-two ilio-femoral grafts without osteotomy and two ilio-femoral grafts with osteotomy-have been excluded owing to incomplete recent information. From these 147 operations there has been one death. This occurred in a child of ten subjected to combined ilio-femoral graft and osteotomy. This case has also been omitted from the results.

Thus 142 cases are available for review-fifty-three ilio-femoral grafts without osteotomy, thirty-four ilio-femoral grafts followed by an osteotomy of the upper end of the femur after a variable interval, and fifty-five ischio-femoral grafts of the Brittain or Foley type.

Of the fifty-three ilio-femoral grafts without osteotomy, nineteen were failures because the grafts failed to fuse at one or other end or became absorbed. In twelve cases a satisfactory extra-articular arthrodesis occurred, with an intact graft fused at both ends but without bony fusion across the joint. In twenty-two cases bony fusion occurred across the joint (Figs. 10 to 14$)$.

VOl. 38 B, NO. 1, FEBruARY 1956 


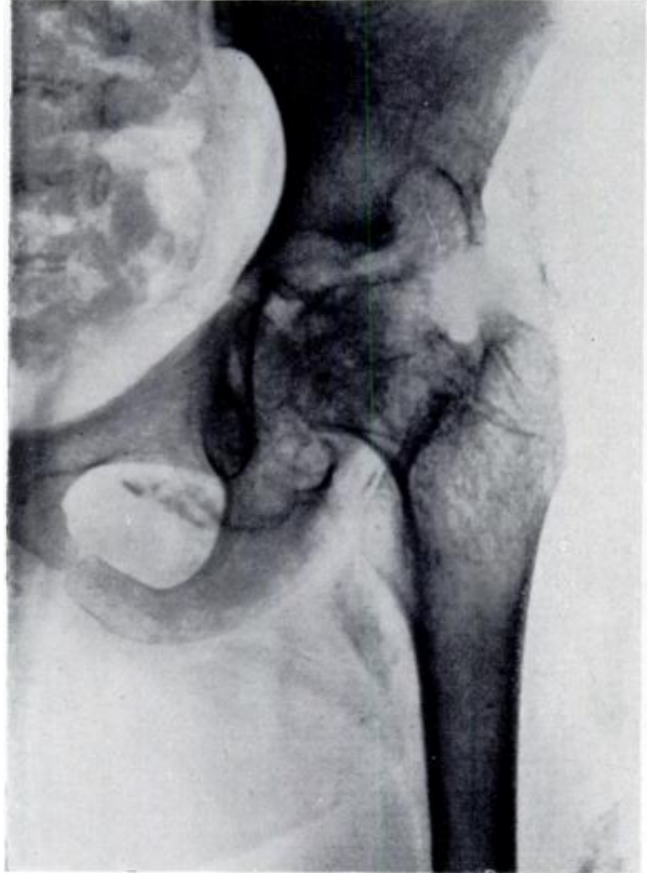

FIG. 10

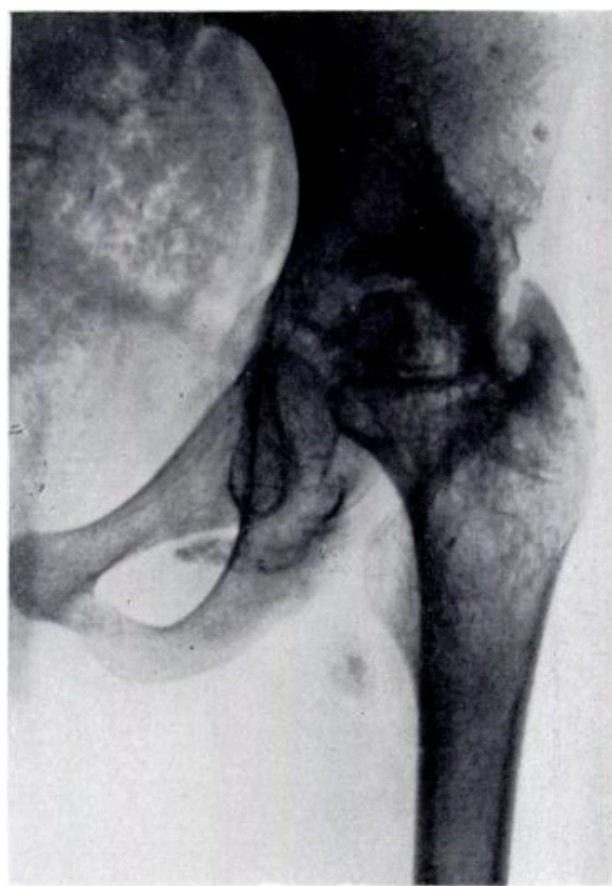

Fig. 11

Case 2. Figure 10-Quiescent tuberculosis of the left hip in a girl of eight. Figure 11-Nineteen months after ilio-femoral graft without osteotomy, showing absorption of the grafts opposite the joint line.

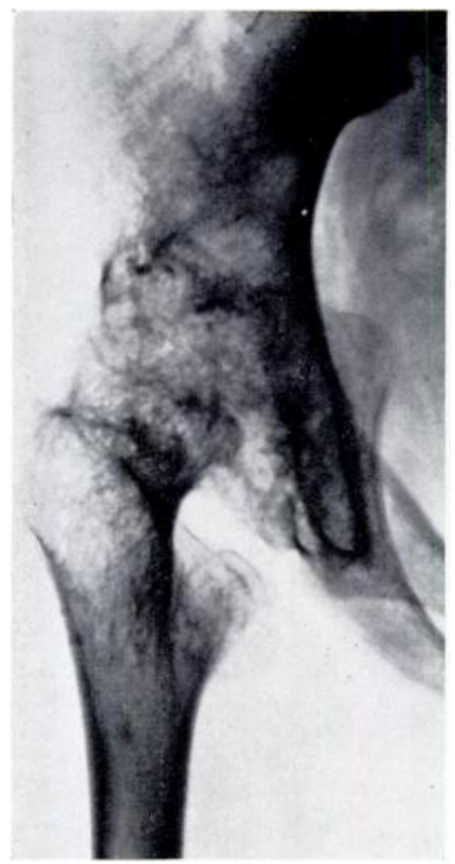

FIG. 12

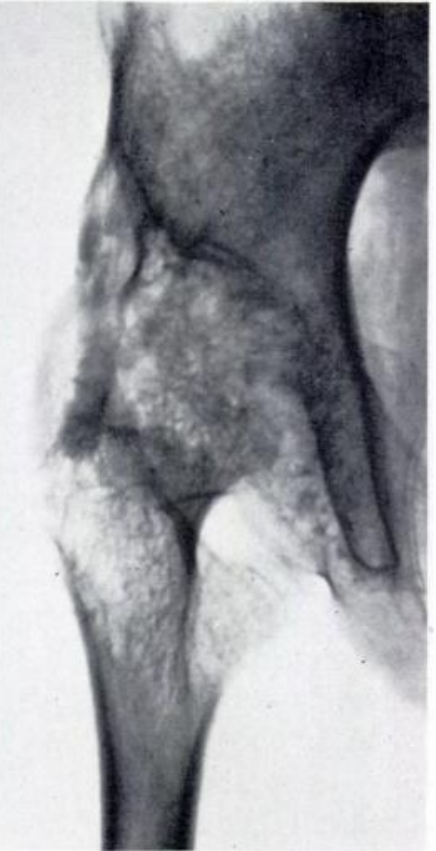

FIG. 13

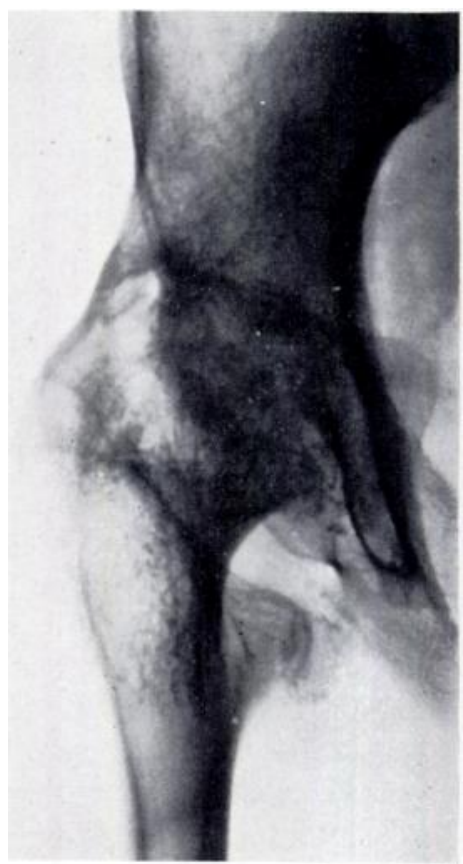

Fig. 14

Case 3. Figure 12-Quiescent tuberculosis of the right hip in a boy of twelve. Figure 13-Four months after ilio-femoral graft without osteotomy. Figure 14-At fifteen months the ilio-femoral grafts fused to the femur and ilium. Bony fusion also appears to be occurring across the joint. 


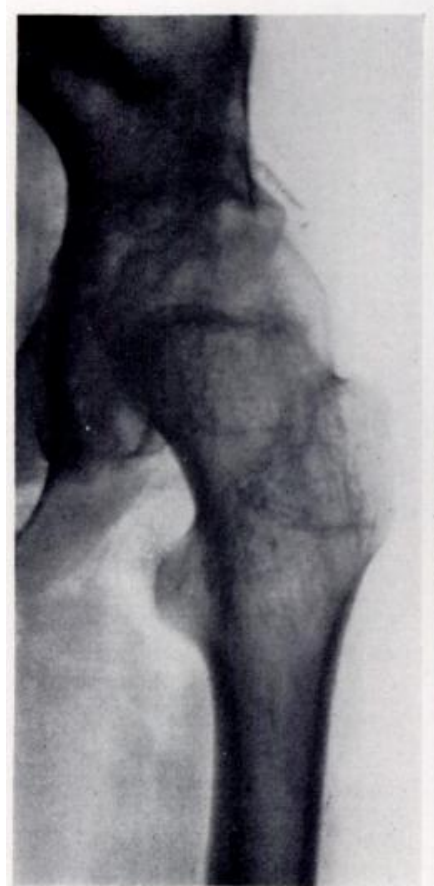

Fig. 15

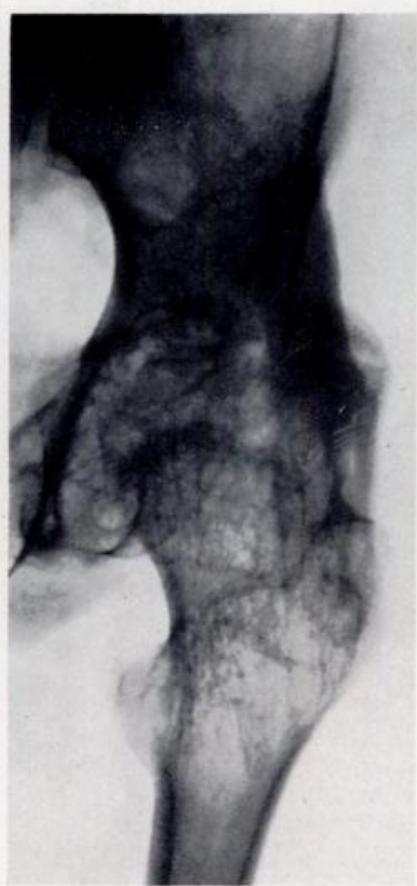

FIG. 16

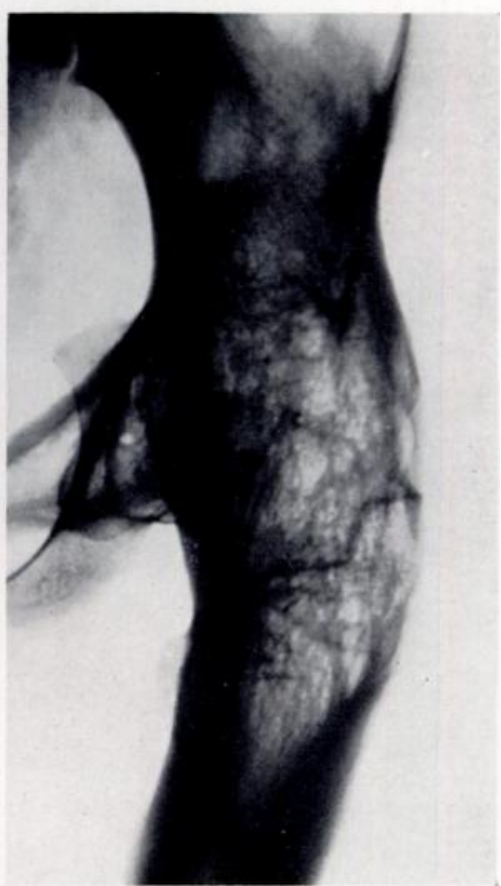

Fig. 17

Case 4. Figure 15-Quiescent tuberculosis of left hip in a girl of twelve. Figure 16-Four months after ilio-femoral graft and immediately before subtrochanteric osteotomy. Figure 17-Twenty-seven months after the graft and twenty-three months after osteotomy, showing bony fusion across the joint. An example of ilio-femoral graft and " early" osteotomy.

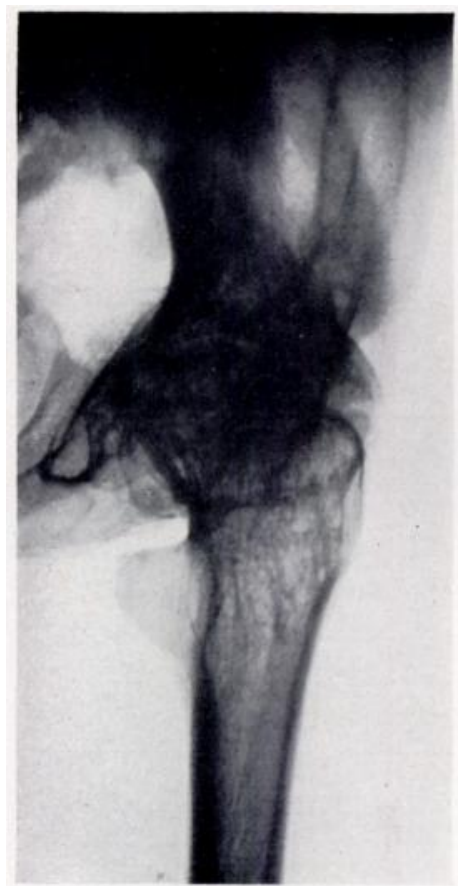

FiG. 18

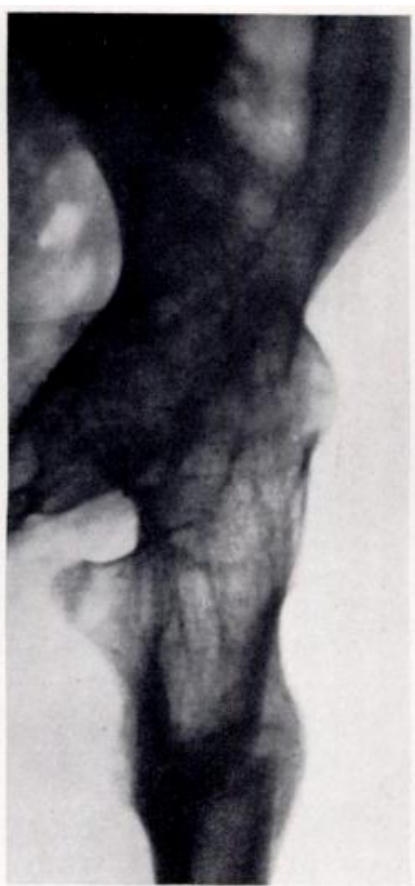

Fig. 19

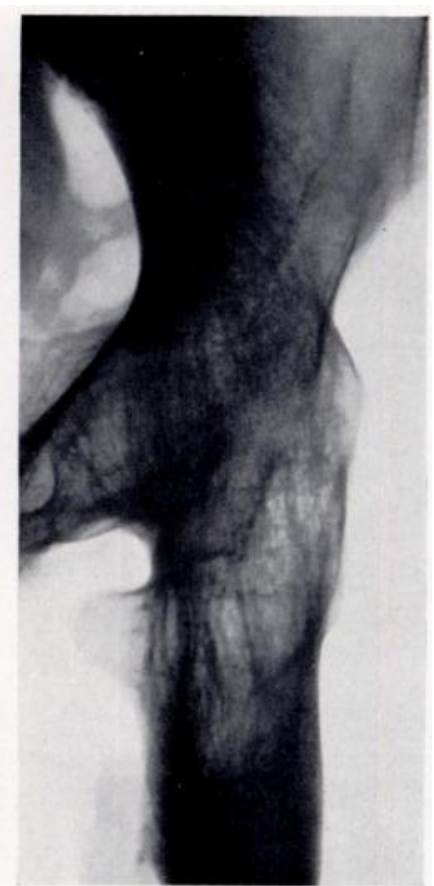

FIG. 20

Case 5. Figure 18-Tuberculosis of the left hip in a girl of sixteen. The radiograph, taken ten years after ilio-femoral graft without osteotomy, shows a gap in the grafts opposite the joint. Figure 19-Four months after subtrochanteric osteotomy to correct flexion, adduction and lateral rotation deformity. Figure 20 Forty-two months after osteotomy, showing sound bony fusion across the joint and of the grafts.

VOL. 38 B, NO. 1, FEBrUARY 1956 


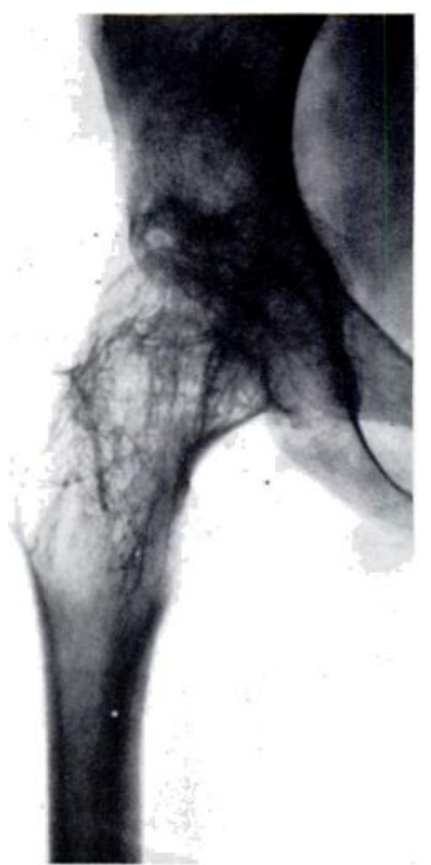

Fig. 21

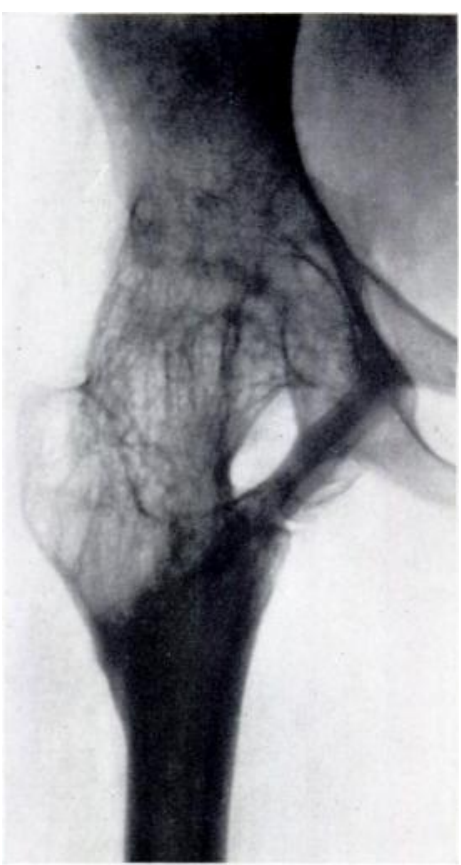

FIG. 22

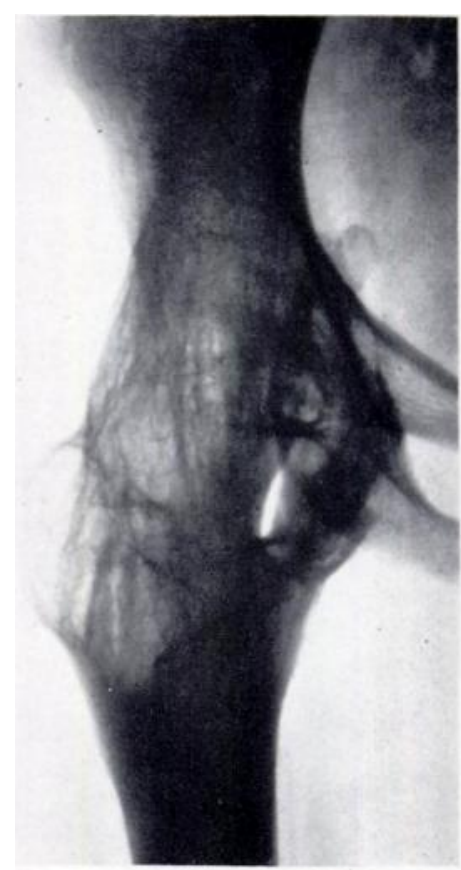

Fig. 23

Case 6. Figure $21-$ Quiescent tuberculosis of the right hip in a girl of eleven. Figure 22-Ten months after ischio-femoral graft by the posterior approach, showing soundly united graft and bony fusion across the joint. Figure 23-Five years after graft showing hypertrophy of the graft, sound bony fusion across the joint and atrophy of the great trochanter and lateral part of the femoral neck due to altered stresses.

Among the thirty-four ilio-femoral grafts followed by an osteotomy at an unspecified interval, there were four failures and four satisfactory extra-articular grafts without bony fusion across the joint. In the remaining twenty-six cases there was bony fusion across the joint (Figs. 15 to 20).

Of the fifty-five ischio-femoral grafts with osteotomy, seven were failures and fifteen were satisfactory grafts without promoting bony fusion across the joint. In thirty-three cases there was bony fusion across the joint (Figs. 21 to 23) (Table I).

TABLE I

Results (All Cases)

\begin{tabular}{|c|c|c|c|c|c|}
\hline \multirow[b]{2}{*}{ Type of operation } & \multirow[b]{2}{*}{$\begin{array}{l}\text { Number } \\
\text { of cases }\end{array}$} & \multirow[b]{2}{*}{ Failure } & \multicolumn{3}{|c|}{ Successful result } \\
\hline & & & $\begin{array}{l}\text { Fused graft } \\
\text { only }\end{array}$ & $\begin{array}{l}\text { Bony fusion } \\
\text { across joint }\end{array}$ & Total \\
\hline Ilio-femoral graft alone & 53 & $19(35 \cdot 8 \%)$ & $12(22.6 \%)$ & $22(41 \cdot 5 \%)$ & $34(64 \%)$ \\
\hline Ilio-femoral graft with osteotomy & 34 & $4(11 \cdot 75 \%)$ & $4(11 \cdot 75 \%)$ & $26(76 \cdot 5 \%)$ & $30(88 \%)$ \\
\hline Ischio-femoral graft & 55 & $7(12 \cdot 7 \%)$ & $15(27 \cdot 2 \%)$ & $33(60 \cdot 0 \%)$ & $48(87 \%)$ \\
\hline
\end{tabular}

Thus thirty-four (64.1 per cent) ilio-femoral grafts without osteotomy, thirty $(88.25$ per cent) ilio-femoral grafts with osteotomy, and forty-eight $(87.2$ per cent) ischio-femoral grafts (Brittain or Foley type) resulted in successful extra-articular or intra-articular arthrodesis.

When the ilio-femoral grafts followed by osteotomy were subdivided into two groups, those with "early" osteotomy to correct pre-existing deformity, and those with "late" osteotomy to correct subsequent deformity, the results were as follows. Seventeen ilio-femoral 


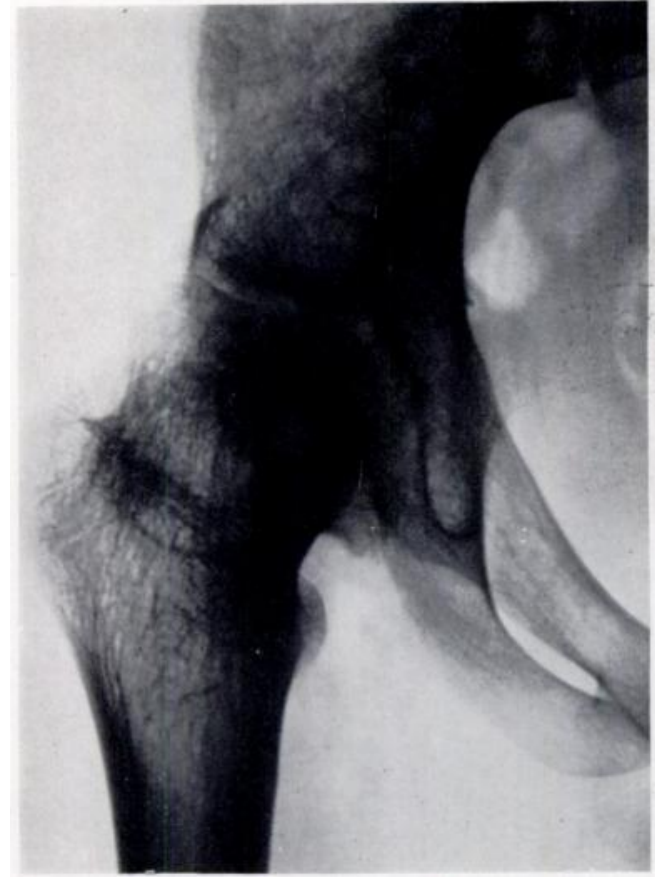

FIG. 24

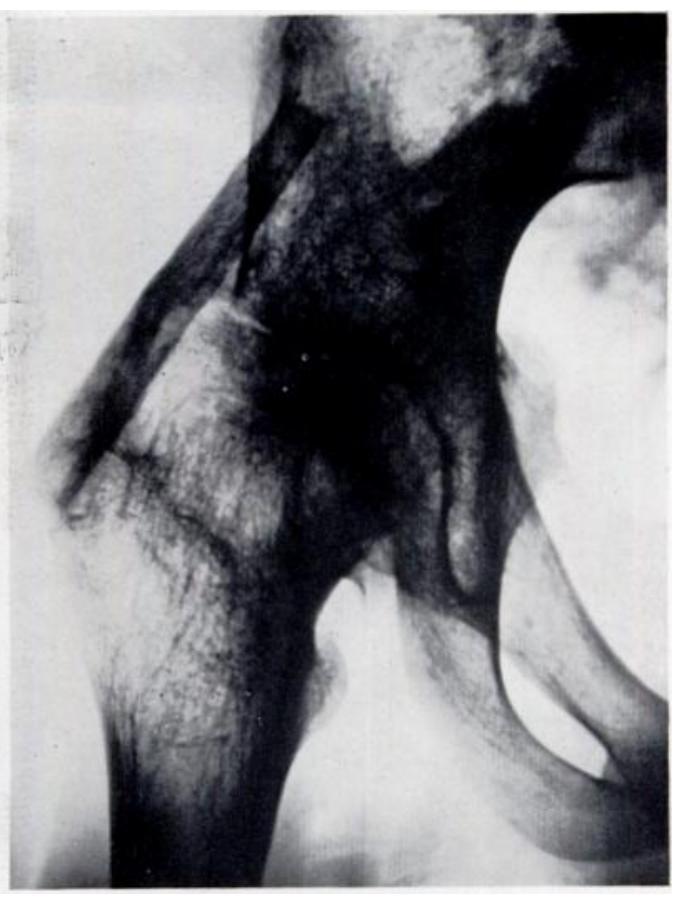

FIG. 25

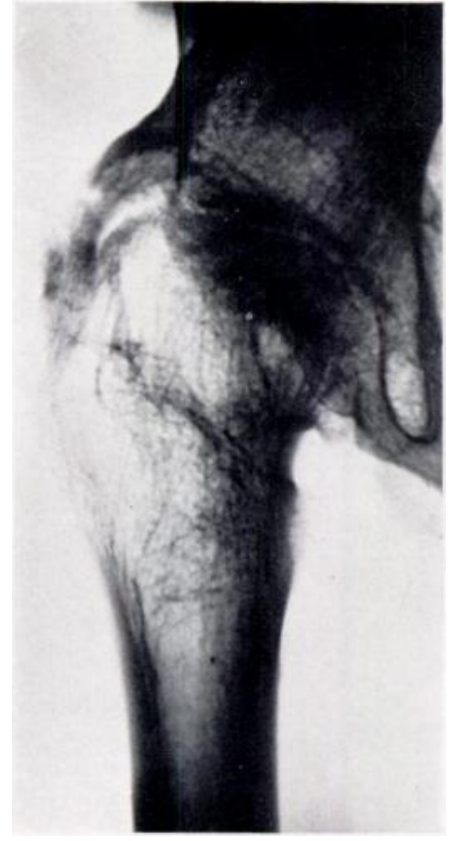

FIG. 26

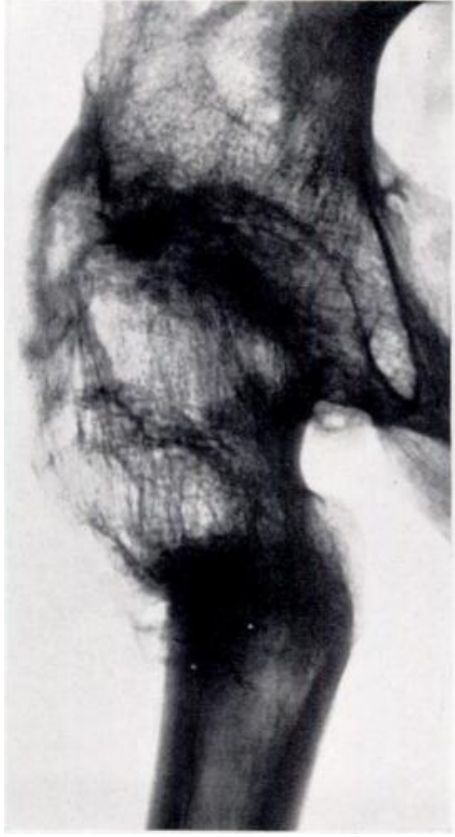

FIG. 27

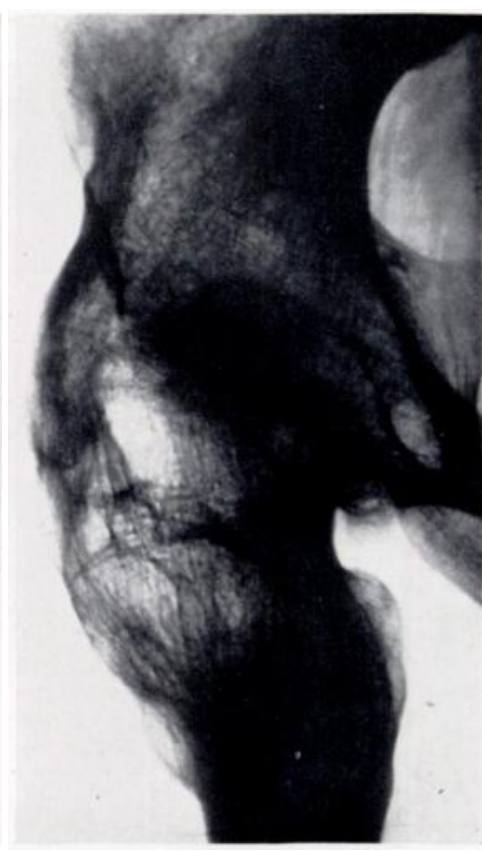

FIG. 28

Case 7-Tuberculosis of right hip, beginning at age three. Figure 24-Radiograph at age fourteen. Quiescent disease with a persistent irregular joint space. Figure 25-Four months after ilio-femoral graft using one piece of tibia. Figure 26-Twenty-eight months after operation showing absorption opposite the joint line, which is still visible. Figure 27-Four months after subtrochanteric osteotomy to correct adduction deformity. Union of the graft and commencing fusion across the joint. Figure 28-Sixteen months after osteotomy, showing hypertrophied ilio-femoral graft and bony fusion across the joint. 


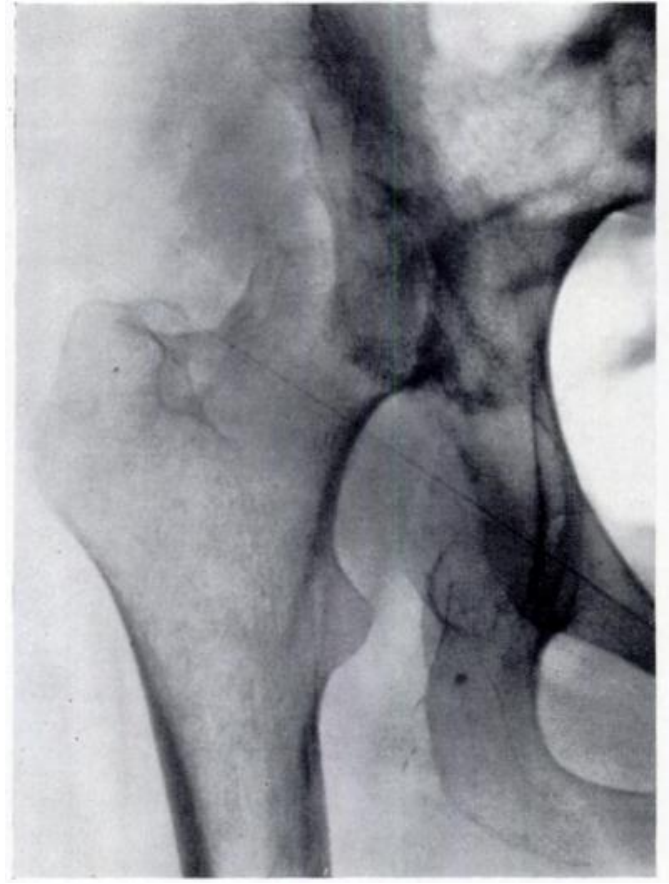

FIG. 29

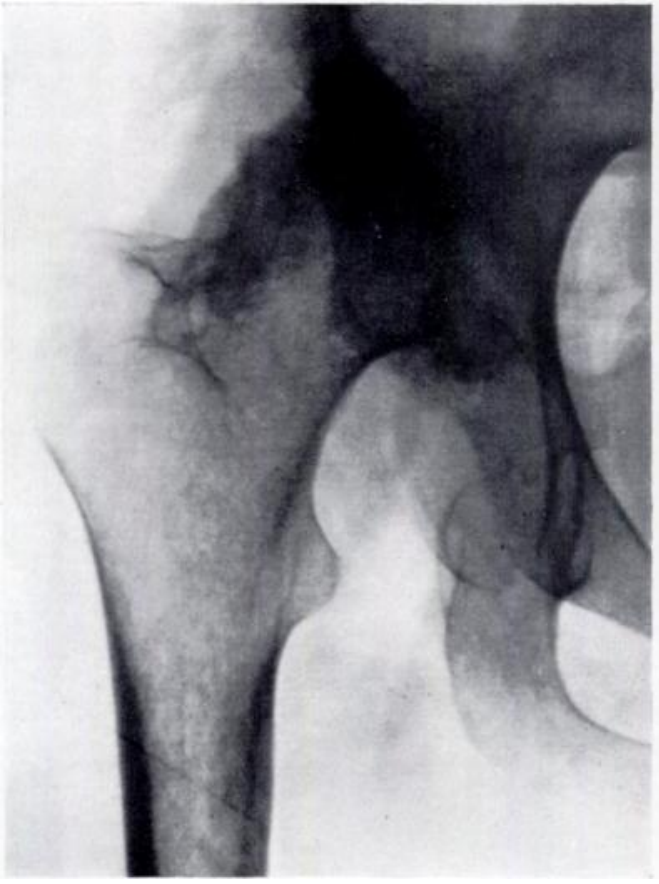

FIG. 30

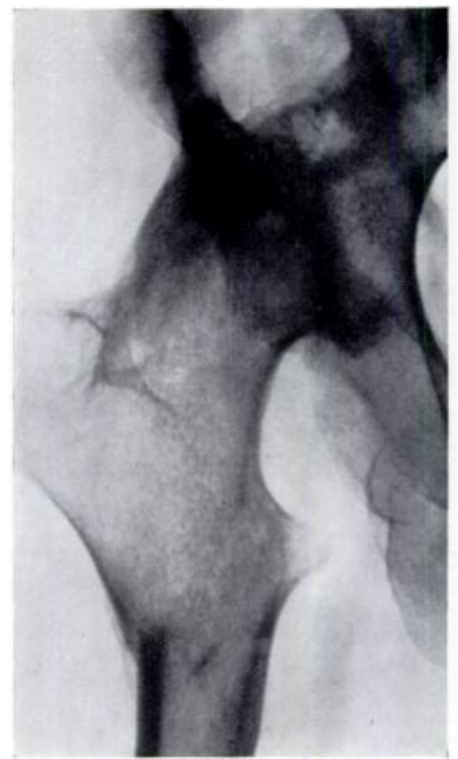

FIG. 31

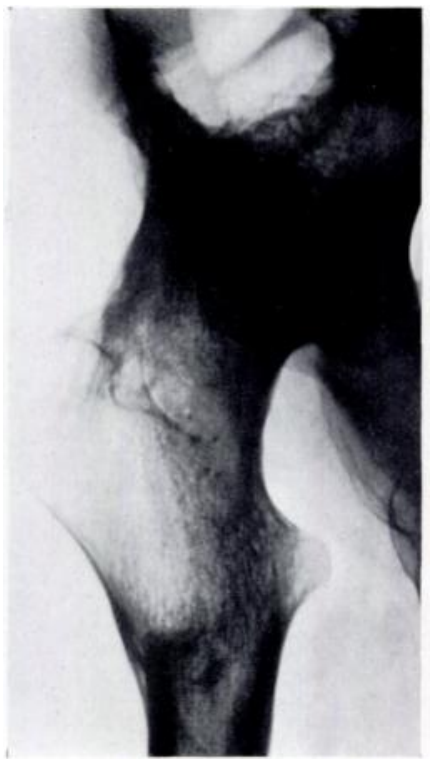

FIG. 32

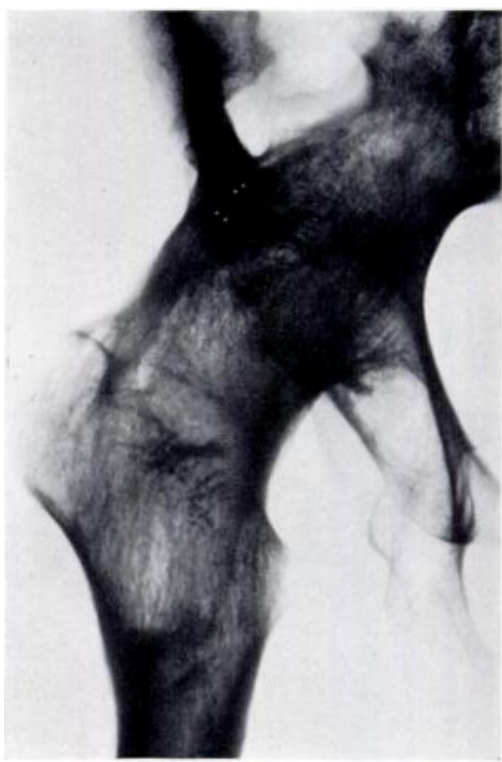

FIG. 33

Case 8-Tuberculosis of the right hip in a woman, beginning at age twenty-two. Figure 29-Radiograph at age twenty-five, showing dislocation of the joint. Figure 30-Six months later and three months after ilio-femoral graft, showing the graft along the upper surface of the femoral neck. Figure 31-Five months after subtrochanteric osteotomy carried out four months after ilio-femoral graft and "early " osteotomy showing satisfactory graft and bony fusion across the joint. Figure 32-Twenty-two months after ilio-femoral graft. Figure 33-Ten years later showing clearly the graft parallel to the upper surface of the femoral neck and sound bony fusion across the joint. 
grafts followed by an " early" osteotomy resulted in three satisfactory grafts without bony fusion across the joint and fourteen cases with bony fusion across the joint; there were no failures. Seventeen ilio-femoral grafts followed by a "late" osteotomy resulted in four failures and one satisfactory graft without bony fusion across the joint; in twelve cases there was bony fusion across the joint (Table II).

TABLE II

Results of Ilio-femoral Graft with Osteotomy Related to Interval between Grafting and Osteotomy

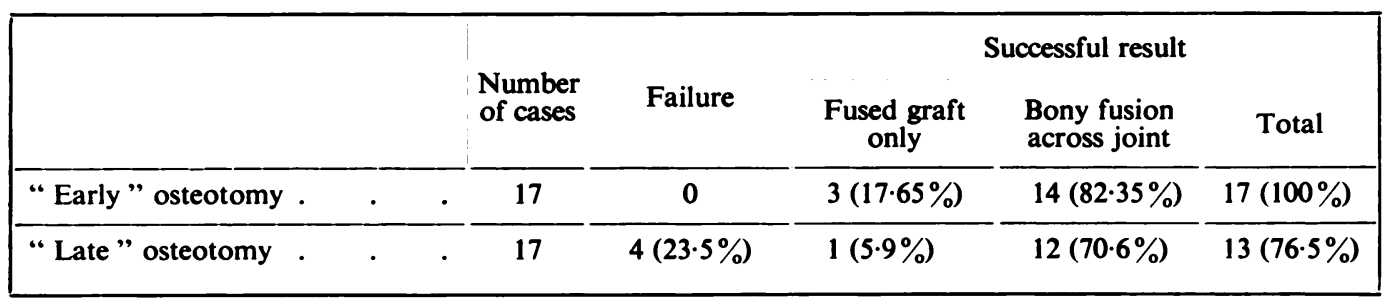

Therefore seventeen (100 per cent) ilio-femoral grafts with "early" osteotomy and thirteen (76.5 per cent) ilio-femoral grafts with "late" osteotomy resulted in successful extra-articular or intra-articular arthrodeses.

The above figures suggest that femoral osteotomy is an important factor in successful extra-articular arthrodesis of a tuberculous hip. Further illustrative radiographs are shown in Figures 24 to 33.

\section{COMMENT}

In the ischio-femoral graft with osteotomy it is advisable to displace the shaft fragment medially to obtain support under the graft and to get a buttress of callus in this situation. In the ilio-femoral graft this medial displacement does not appear to be so important, whether the osteotomy is carried out early or late. If, however, there has been little destruction of the femoral neck medial displacement of the femoral shaft produces better alignment. It also reduces the shearing strain on the graft and the joint.

From these results it appears that, so long as an osteotomy of the upper end of the femur is performed, it does not matter, apart from the site of disease, whether an ilio-femoral or ischio-femoral graft is carried out. The ischio-femoral arthrodesis by the Brittain or Foley technique requires only one operation and there is no doubt, at least in children, that fusion can be surprisingly rapid. Against this, the operation - and particularly the Foley modificationis more severe than either an ilio-femoral graft or a subtrochanteric or intertrochanteric osteotomy.

\section{SUMMARY}

1. One hundred and forty-two cases of extra-articular arthrodesis of quiescent tuberculous hips with fibrous ankylosis have been reviewed.

2. The methods used were the ilio-femoral graft with and without osteotomy, and the ischio-femoral graft by the Brittain or Foley technique.

3. Success occurs more frequently when the grafting operation is combined with or followed by a femoral osteotomy.

4. It is suggested that this success is due largely to the increased immobilisation afforded by the osteotomy.

5. It appears that equally good results can be obtained with either an ilio-femoral or an ischio-femoral graft in these cases provided that an upper femoral osteotomy is also carried out, preferably at or soon after the grafting operation.

6. An upper femoral osteotomy will frequently convert an unsuccessful extra-articular hip graft into a successful one without further grafting.

VOL. 38 B, NO. 1, FEBRUARY 1956 


\section{REFERENCES}

Brittain, H. A. (1941): Ischiofemoral Arthrodesis. British Journal of Surgery, 29, 93.

Brittain, H. A. (1942): Architectural Principles in Arthrodesis. Edinburgh: E. \& S. Livingstone.

Brittain, H. A. (1948): Ischio-femoral Arthrodesis. Journal of Bone and Joint Surgery, 30-B, 642.

Cholmeley, J. A., and NANGle, E. J. (1951): Ischio-femoral Arthrodesis by the Posterior Approach. Journal of Bone and Joint Surgery, 33-B, 365.

Foley, W. B. (1949): Ischio-Femoral Arthrodesis of the Hip by Posterior Open Approach. Journal of Bone and Joint Surgery, 31-B, 222.

MiLch, H. (1947): Osteotomy of the Long Bones. Oxford: Blackwell Scientific Publications Ltd.

Stevenson, F. H. (1954): The Chemotherapy of Orthopaedic Tuberculosis. Journal of Bone and Joint Surgery, 36-B, 5.

Stevenson, F. H. (1954): Bone and Joint Tuberculosis: The Value of Antibiotics. In British Surgical Practice. Surgical Progress 1954. Under the General Editorship of Sir Ernest Rock Carling and Sir James Paterson Ross. London: Butterworth \& Co. (Publishers) Ltd.

Trumble, H. C. (1932): A Method of Fixation of the Hip Joint by Means of an Extraarticular Bone Graft. Australian and New Zealand Journal of Surgery, 1, 413.

Trumble, H. C. (1937): Fixation of the Hip-Joint by Means of an Extra-articular Bone-Graft: Late Results. British Journal of Surgery, 24, 728.

VAN Gorder, G. W. (1949): The Trumble Operation for Fusion of the Hip. Journal of Bone and Joint Surgery, 31-A, 717.

Wilkinson, M. C. (1947): Intertrochanteric Osteotomy for the Treatment of Tuberculosis of the Hip. Proceedings of the Royal Society of Medicine (Section of Orthopaedics), 40, 238. 